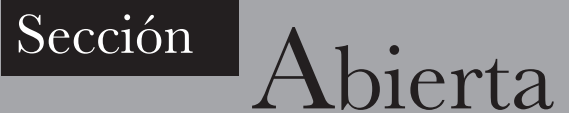

\title{
VULNERABILIDAD SOCIAL DE LAS MUJERES Y LA POBLACIÓN INDÍGENA EN OAXACA, 2005: RESTRICCIONES Y ESTRATEGIAS
}

\author{
Rafael G. Reyes Morales \\ Alicia Sylvia Gijón Cruz
}

\begin{abstract}
Resumen: Los ejes de la discusión de la vulnerabilidad social de las mujeres y los indígenas en el estado de Oaxaca son: la exclusión de éstos beneficios de las políticas públicas de bienestar; el poder adquisitivo; las estrategias para superar la condición de vulnerabilidad. Los hallazgos del artículo contradicen la situación de relativa precariedad de los hogares con jefatura femenina reportada por la literatura especializada. La migración aparece como un instrumento que contribuye a la equidad de género y permite elevar la escolaridad de la población indígena. Finalmente, la población indígena es el grupo de población prácticamente excluido de las políticas de bienestar y tiene el menor poder adquisitivo, mientras que las mujeres indígenas constituyen el grupo que menos beneficios recibe de la política pública de educación.

Palabras clave: Vulnerabilidad social, género, población indígena, migración interna e internacional.
\end{abstract}

Enviado a dictamen: 14 de marzo de 2007. Aprobación: 08 de agosto de 2007.

Rafael G. Reyes Morales, doctor en Ciencias por el Instituto Tecnológico de Oaxaca, Migración y desarrollo, correo electrónico: ragaremo@gmail.com. Alicia Sylvia Gijón Cruz, maestra en Ciencias y candidata a doctora en cien cias por el Instituto Tecnológico de Oaxaca, profesora de la Facultad de Ciencias Químicas, Universidad Autónoma Benito Juárez de Oaxaca, temas de especialización: Migración internacional, roles de la mujer y bienestar, correo electrónico: gijoncruz@gmail.com.
Abstract: The central points of discussion on social vulnerability of women and indigenous population in Oaxaca State include: Exclusion from policies affecting welfare benefits; purchasing power; and strategies for overcoming the condition of vulnerability. The findings of this article contradict the female-headed households' state of relative precariousness as reported in case specific literature. Migration appears as an instrument contributing to gender equity and allows for increased schooling in indigenous population. Furthermore, the indigenous population is almost excluded from welfare polices and has lower purchasing power, while indigenous women represent the most excluded group from public polices on education.

Key words: Social vulnerability, gender, indigenous population, internal and international migration.

\section{Introducción}

E n este artículo se evalúa, con base en 570 municipios del estado de Oaxaca y de manera específica una comunidad zapoteca, la vulnerabilidad social de las mujeres y los indígenas en el estado de Oaxaca; asimismo, se analizan las estrategias que adoptan estos actores sociales para superar su condición de exclusión e inseguridad. Las unidades de análisis en el ámbito municipal son las siguientes: para las mujeres, se consideran los hogares con jefatura femenina, la 
población femenina y las mujeres indígenas; para los indígenas, la población indígena municipal. Los hogares con jefatura femenina son aquellos en los hace falta el esposo o cónyuge y por lo tanto la jefatura corresponde normalmente a la madre. Cuando está ausente también la madre, entonces una hija u otra mujer pueden asumir la jefatura. En cambio, los hogares con jefatura masculina se refieren a aquellos hogares en los que normalmente están presentes los dos cónyuges y con menos frecuencia incluyen hogares en donde la esposa o cónyuge está ausente. La principal característica de este tipo de hogares consiste en asumir que la jefatura corresponde al esposo o cónyuge masculino.

Los ejes de análisis son: la exclusión de mujeres e indígenas de los beneficios de las políticas públicas — con relación a la vivienda, educación y salud-; el poder adquisitivo y las estrategias para superar estas restricciones (tamaño de los hogares, migración interna e internacional y educación). Se pretende mostrar de manera objetiva la vulnerabilidad social de las mujeres y los indígenas en el estado de Oaxaca en tres niveles de análisis. Primero, se evalúan las condiciones socioeconómicas de las mujeres y los indígenas a través de las variables seleccionadas para las unidades de análisis. Segundo, se utiliza el análisis de correlación de Pearson para medir la fuerza de asociación entre las unidades de análisis y las variables seleccionadas. ${ }^{1}$ Finalmente, en el artículo se analiza, a través de una comunidad zapoteca, las estrategias de largo plazo adoptadas para superar la condición de exclusión tanto de parte del Estado mexicano como de los mismos hogares hacia las mujeres.

En este ensayo los elementos considerados en las políticas públicas de bienestar son: vivienda (servicios básicos), educación, salud y equipamiento urbano. El bajo poder adquisitivo de los actores socialmente vulnerables es el resultado del modelo de desarrollo nacional el cual no tiene la capacidad para ofrecer suficientes empleos bien remunerados para la creciente población. En particular, podemos referirnos a la política salarial del Estado mexicano que mantiene bajos salarios mínimos.
Entenderemos por vulnerabilidad social de los grupos de la población de un estado o país en un período determinado, la condición que resulta de la exclusión de los beneficios de las políticas públicas de bienestar y del insuficiente acceso a los recursos vitales, bienes y servicios (Rodríguez Vignoli, 2004; Busso, 2001). Otra condición de la vulnerabilidad se refiere a la dificultad de los grupos excluidos - que llamaremos en adelante vulnerables - para dar respuesta a los efectos de eventos adversos, como crisis económicas nacionales, catástrofes naturales y exclusión de las políticas públicas (Pizarro, 2001: 11-12; Salgado de Zinder et al., 2007: 8-10; Rodríguez Vignoli, 2004). Tal debilidad de respuesta está vinculada a la escasez de activos y al bajo poder adquisitivo que mantiene a los miembros de los hogares en una condición de vulnerabilidad. Como consecuencia, bajo condiciones adversas, el consumo de alimentos puede llegar a reducirse por debajo del nivel de subsistencia o los hijos pueden abandonar la escuela para trabajar y ayudar en el gasto familiar. En ocasiones, ni siquiera la venta de los escasos activos familiares llega a ser suficiente para hacer frente a los gastos por enfermedades, defunción o problemas legales. En este contexto, aparece la migración como una opción viable para obtener ingresos que ayudarán a superar la crisis en menor tiempo e incluso a mejorar las condiciones de vida.

Las políticas públicas de bienestar y el índice de marginación proveen un marco analítico más adecuado para evaluar la vulnerabilidad social a diferencia del concepto vago de exclusión social y el complejo concepto de marginalidad económica (Cortés, 2006: 71-84).

\section{La población femenina y población indígena en el estado de Oaxaca, 2005}

Las mujeres constituyen en promedio $52.6 \%$ de la población de los municipios de la entidad según datos del conteo de población y vivienda 2005 del Instituto Nacional de Estadística, Geografía e Informática (INEGI). 
Esta proporción de la población femenina se sostiene incluso en los demás municipios cuyo porcentaje de población indígena es igual o mayor a la media de los municipios. El porcentaje de población femenina por municipio sigue aproximadamente una distribución normal estándar aunque se presenta una fuerte concentración de municipios con valores cercanos a la media (figura 1). Así, son pocos los municipios que tienen menos de la mitad o más de $55 \%$ de población femenina. La mayoría de los municipios que tienen más hombres que mujeres se encuentran en el intervalo de 116 a 3,187 habitantes, mientras que la mayoría de los municipios con porcentajes de población femenina entre 55 y 60\% tienen entre 235 y 2,298 habitantes. Estos municipios con valores extremos de población femenina normalmente son muy pequeños (menores a 500 habitantes) o bien están formados por muchas comunidades muy pequeñas. En los dos casos la estructura demográfica de las comunidades es muy sensible a la pérdida de población como resultado de la migración. Las mujeres oaxaqueñas, en 2004, tenían una esperanza de vida de 76.41 años y estaban 1.22 años por debajo de la media nacional, pero 5.21 años arriba de la esperanza de vida de los hombres oaxaqueños ${ }^{2}$. La población indígena en el estado de Oaxaca tanto de hombres como de mujeres está a un año aproximadamente por debajo de la esperanza de vida de la población total de hombres y mujeres. ${ }^{3}$ Las mujeres oaxaqueñas constituyen la mayoría de la población a partir del grupo quinquenal de 25 a 29 años hacia arriba de la pirámide de edades. En el ámbito nacional la población femenina sigue esta tendencia a partir del quinquenio de 30 a 34 años de edad. Es decir, el promedio de edad de las mujeres oaxaqueñas es menor que el de las mujeres a nivel nacional. ${ }^{4}$

La población indígena por municipio es en promedio de $40.6 \%$ y sigue una distribución en forma de U en la cual la mayoría de los municipios se concentra en los extremos (figura 2). En el extremo izquierdo está un grupo de municipios que tienen menos de 10\% de población indígena y representan $38.6 \%$ del total. La mayoría de estos municipios tienen menos de 2,000 habitantes, sin embargo, este grupo también incluye dos de los municipios más poblados de la entidad (Oaxaca de Juárez con 265,006 habitantes y Salina Cruz con 76,219 habitantes). En el extremo derecho se encuentra el grupo de municipios con más de $90 \%$ de población indígena, los cuales hacen $22.6 \%$ del total. La gran mayoría de estos municipios tienen una población menor a 4,000 habitantes y el municipio más grande Huautla de Jiménez, tiene 31,829 habitantes. En una posición intermedia se encuentran otros dos de los municipios más grandes de la entidad. San Juan Bautista Tuxtepec, el segundo más grande, tiene $16.4 \%$ de población indígena, mientras que Juchitán de Zaragoza, el tercer municipio, alcanza $65.8 \%$.

El índice "marginación 2005" constituye una variable construida por el Consejo Nacional de Población (CONAPO), mediante el método de componentes principales, y agrupa indicadores que miden la exclusión de la población de los servicios básicos de la vivienda (agua entubada, energía eléctrica y drenaje), del equipamiento urbano (municipios menores de 5,000 habitantes) y de los servicios educativos (educación primaria y analfabetismo). Asimismo, considera la población ocupada de bajo poder adquisitivo (que gana hasta dos salarios mínimos) y viviendas con pisos de tierra. Este último indicador ya forma parte de la política pública de bienestar del gobierno del estado de Oaxaca, la cual se aplica de manera clientelar. ${ }^{5}$ Por lo tanto, este índice se puede utilizar como una medida multivariante de exclusión de las políticas públicas de vivienda, equipamiento urbano y educación comunes a las áreas rurales y urbanas, pero también como una medida del bajo poder adquisitivo. Con el propósito de separar los efectos de la ausencia de los servicios básicos de la vivienda y la permanencia de los pisos de tierra, se cálculo un índice de vivienda. Se siguió estrictamente el procedimiento de cálculo del CONAPO que incluye la aplicación del método de componentes principales para la construcción del índice y estratificación óptima de Danelius y Hodges, según 
Martínez Alonso (2004: 60-90) y CONAPO (2006: 174-175). El índice de vivienda obtenido para los 570 municipios captura $81.482 \%$ de la variabilidad total en un solo factor. La escala ordinal del índice conforme decrece y toma valores cada vez más negativos denota viviendas con servicios básicos y sin pisos de tierra. Por el contrario, cuando el índice de vivienda adopta valores positivos cada vez más grandes indica viviendas precarias. En el ámbito estatal, este índice se estimó calculando el promedio de todos los municipios.

La población indígena es el sector de la población más vulnerable. En particular, la mujer indígena se encuentra en clara desventaja respecto a la población total y a los hogares con jefatura femenina, cuando se considera el índice de marginación, el índice de vivienda, el analfabetismo en forma separada y la escolaridad promedio (cuadro 1). Sin embargo, son menos frecuentes los hogares indígenas con jefatura femenina ( debido a la fuerte cohesión de sus hogares y comunidades. Los municipios indígenas - aquellos que tienen un porcentaje de población indígena mayor al promedio del total de los municipios de la entidad (mayor a $40.6 \%$ ) - son de muy alta marginación, mientras que los hogares con jefatura femenina y con jefatura masculina en la entidad son de alta marginación. ${ }^{6}$ Esto implica que la población de los municipios indígenas se encuentra en desventaja con relación a su poder de adquisitivo, equipamiento urbano de sus comunidades (localidades con menos de 5,000 habitantes), acceso a la educación y a los servicios básicos de la vivienda.

Para poder evaluar la vulnerabilidad social, fueron considerados dos indicadores no incluidos en el índice de marginación: ser excluidos de los servicios de salud (porcentaje de población sin derechohabiencia a servicios de salud) y de la posesión de aparatos electrodomésticos modernos (televisor, refrigerador, lavadora y computadora). Se construyó también un índice de electrodomésticos siguiendo el procedimiento del índice de vivienda tanto en los municipios como en el estado. En este caso el índice tiene $45.075 \%$ de la variabilidad total en un solo factor. La población indígena también se encuentra en desventaja en cuanto a la posesión de electrodomésticos y acceso a los servicios de salud públicos y privados.

Las mujeres indígenas constituyen sin duda el grupo de la población oaxaqueña excluido de la política pública de educación: ellas tienen los mayores niveles de analfabetismo y los más bajos niveles de escolaridad (cuadro l).

El tamaño promedio de los hogares en los municipios indígenas (4.57\%) es mayor que el de la entidad; esto incluye a la población total (4.36\%), los hogares con jefatura femenina $(4.12 \%)$ y los hogares con jefatura masculina (4.53\%). Esta condición agrava la precariedad de sus miembros asociada a sus bajos ingresos. La población de los municipios indígenas también presenta patrones de migración diferentes a los de la entidad. En primer lugar, recurren menos a la migración interna y prácticamente no existe diferencia en la estructura por sexo de los flujos de migrantes. En segundo lugar, la migración internacional es mayor que la producida en la entidad (0.31\%). Esto se puede atribuir al hecho de que las remesas internacionales que reciben los hogares expulsores de población normalmente son mayores que las remesas nacionales y, por lo tanto, las primeras impactan más en el poder adquisitivo (Reyes Morales et al., 2005).

La estrategia de los indígenas de mantener un tamaño de hogar relativamente grande - ligado a un mayor número de hijos - que el promedio de la entidad, constituye un recurso para asegurar mano de obra en su unidad de producción familiar. Asimismo, tal tamaño de hogar representa una reserva de mano de obra para obtener ingresos a través del trabajo asalariado, la migración interna e internacional. Sin embargo, con los bajos ingresos de estos hogares, se reduce el consumo de alimentos y de otros bienes y servicios. Esta situación se agrava todavía más durante los períodos cuando no fluyen las remesas; cuando ocurren pérdidas de cosechas; cuando aqueja una enfermedad grave a un miembro del hogar o se enfrentan problemas legales. 
La vulnerabilidad de las mujeres normalmente se analiza a partir de los hogares con jefatura femenina en los cuales no existe el esposo o cónyuge. La gran mayoría de los estudios coinciden en que este tipo de hogares poseen menos bienes, reciben menores ingresos y tienen viviendas de menor calidad que los hogares con jefatura masculina. ${ }^{7}$ Sin embargo, en el estado de Oaxaca los hogares con jefatura femenina se encuentran en mejor posición que los hogares con jefatura masculina y sobre todo de la población indígena. Los beneficios que reciben de las políticas públicas —de vivienda, educación y salud -, los hogares con jefatura femenina tienen una relación directa con su ubicación - en mayor proporción-en localidades con características urbanas (mayores a 5,000 habitantes). Un menor tamaño de los hogares con jefatura femenina y una mayor escolaridad de sus miembros permiten que este tipo de hogares obtengan mejores salarios y sostengan un mayor poder adquisitivo per cápita. Otra estrategia de estos hogares para incrementar sus ingresos es la migración, ya que éstos presentan flujos migratorios tanto internos como internacionales (cuadro 1). Queda pendiente la discusión de los aspectos organizativos de estos hogares referentes a la administración del presupuesto familiar y sus iniciativas para emprender negocios con las remesas de la migración (Morris y Winter, 1996; Portes y Guarnizo, 1991); estas tareas pendientes seguramente abren otras importantes vetas para profundizar en el análisis de las estrategias de los hogares con jefatura femenina para superar sus restricciones y elevar su calidad de vida.

\section{¿Vulnerabilidad de los hogares con jefatura femenina?}

Se utilizó el coeficiente de correlación de Pearson (Nourisis, 1993) para medir el grado de asociación de los hogares con jefatura femenina con la población indígena, las características socioeconómicas de los hogares y la migración. Para comprender mejor los resultados se calculó también el coeficiente de correlación para los hogares con jefatura masculina y con la población femenina total. De esta manera, podemos medir la relación estadística (probabilística) entre estos hogares, sus condiciones socioeconómicas, sus niveles de exclusión de las políticas públicas de bienestar (vivienda, educación y salud), así como del consumo de bienes modernos. Esta metodología constituye una propuesta para analizar la vulnerabilidad social tanto en áreas rurales como urbanas con un enfoque interdisciplinario y multidimensional. Muchos de los trabajos que están orientados a medir la vulnerabilidad social intentan prescribir o justificar políticas públicas (Álvarez Ayuso y Cadena Vargas, 2006; Rodríguez Vignoli, 2004; Domill, Frenkel y Mauricio, 2003; Pizarro, 2001: 43-51; Kaztman, 2000). En nuestro caso, abordamos las políticas públicas de bienestar a partir de los resultados reportados por el conteo de población y vivienda 2005 (INEGI, 2006). Los niveles de ingreso (CONAPO, 2006) y el consumo de electrodomésticos (INEGI, 2006) constituyen indicadores del poder adquisitivo de los grupos vulnerables y de los efectos del modelo de desarrollo nacional. Por lo tanto, el coeficiente de correlación permite establecer relaciones causales entre actores o grupos vulnerables (indígenas, mujeres indígenas, hogares con jefatura femenina) y los factores determinantes (condiciones y estrategias). En cambio, los índices de vulnerabilidad social solamente se limitan a elaborar escalas de medición (Álvarez Ayuso y Cadena Vargas, 2006; Kaztman, 2000).

Observamos que la población indígena guarda una correlación inversa con los hogares con jefatura femenina (coeficiente con signo negativo). Es decir, conforme se incrementa el porcentaje de población indígena por municipio disminuye el número de hogares con jefatura femenina (cuadro 2). Este resultado es consistente con los valores promedio del cuadro uno. Las sociedades indígenas sostienen valores que favorecen la preservación de los hogares con jefatura masculina, los cuales cuentan generalmente con la presencia de la esposa o cónyuge. No se detectó correlación entre la población indígena y la población femenina. Este resultado indica que la concentración de población femenina en los municipios 
con población indígena del estado de Oaxaca no guarda una relación estadísticamente consistente.

Los hogares con jefatura femenina como la población femenina (total) están inversamente correlacionados con el índice de marginación, mientras que los hogares con jefatura masculina se correlacionan de manera directa con dicho índice (coeficiente con signo positivo). Estas relaciones contrastantes arrojan un resultado realmente sorprendente. Los hogares con jefatura femenina y las mujeres, en general, se encuentran menos excluidos de las políticas públicas de vivienda, educación y equipamiento urbano, así también tienen menos bajos salarios que los hogares con jefatura masculina y en consecuencia que los hombres en general. Si revisamos la correlación específica con los servicios básicos de la vivienda, el acceso a la educación primaria y el analfabetismo, encontramos que el análisis descriptivo del cuadro uno no es siempre coherente con los resultados del análisis de correlación (cuadro 2). No existe correlación significativa entre el analfabetismo y los tres grupos de población considerados en esta sección (los hogares con jefatura femenina, hogares con jefatura masculina y población femenina). Se puede decir que el analfabetismo no tiene una relación causal con el tipo de jefatura en el hogar o con el género sino más bien es consecuencia de otros factores que no fueron considerados en el análisis. Lo mismo ocurre con la población sin derechohabiencia a servicios de salud públicos y privados que tampoco presenta correlación estadísticamente significativa con los tres grupos de población. En cambio, los hogares con jefatura femenina sí guardan una correlación directa con el grado promedio de escolaridad y una correlación inversa con el índice de vivienda al igual que la población femenina. Este hallazgo soporta los resultados del cuadro uno en lo referente a que los hogares con jefatura femenina tienden a tener una mayor escolaridad y sus viviendas tienen más servicios básicos y son menos frecuentes los pisos de tierra que en los hogares con jefatura masculina. La interpretación de la correlación con el índice de vivienda no es obvia ya que éste se construyó con indicadores que denotan viviendas sin servicios básicos y con pisos de tierra. Al respecto, fue necesario realizar el análisis de regresión simple para poder explicar cabalmente el significado del signo del coeficiente de correlación. La figura tres muestra que conforme se incrementan los hogares con jefatura femenina disminuye el índice de vivienda; es decir, disminuyen las viviendas sin servicios básicos y con pisos de tierra. Los hogares con jefatura masculina y la población indígena presentan un comportamiento inverso al de los hogares con jefatura femenina. Considerando la estratificación de la escala del índice de vivienda, tenemos que los valores positivos corresponden a las más bajas calificaciones y los valores más negativos a las calificaciones más altas. Así, podemos afirmar que los hogares con jefatura masculina se encuentran en mejor posición que la población indígena con respecto al índice de vivienda.

Asimismo, es menos probable que los hogares con jefatura femenina tengan bajo poder adquisitivo. En el cuadro dos aparecen los coeficientes de correlación de estos hogares con la población de bajo poder adquisitivo y con el índice de electrodomésticos. El coeficiente de correlación con los electrodomésticos modernos es positivo (correlación directa), mientras que el coeficiente de correlación con la población de bajo poder adquisitivo es negativo (correlación inversa), como se esperaba. La interpretación es que los hogares con jefatura femenina tienen mayor poder de compra que los hogares con jefatura masculina, lo cual se refleja en un mayor consumo de aparatos electrodomésticos. La población femenina se comporta en forma muy similar a los hogares con jefatura femenina, excepto que no necesariamente tiene mayor poder adquisitivo que los hogares con jefatura masculina ni concentra menos población indígena que los hogares con jefatura femenina.

Hasta aquí podemos establecer que los hogares con jefatura femenina son menos marginados que los hogares con jefatura masculina por estar menos excluidos de las políticas públicas de educación y vivienda, 
y por tener mayor poder adquisitivo. Los hogares con jefatura femenina tienden a concentrar, además, menos población indígena. La población femenina coincide en varias de las correlaciones de los hogares con jefatura femenina. Esto se debe seguramente a que incluye a todas las mujeres entre las que se encuentran aquellas de los hogares con jefatura femenina y masculina. En este sentido, se puede afirmar que la población femenina es redundante, en cierto grado, con los dos tipos de hogares. Los hogares con jefatura masculina son más marginados y concentran mayor población indígena que los hogares con jefatura femenina.

La correlación inversa de los hogares con jefatura femenina con el índice de marginación sugiere que estos hogares realizan un mayor esfuerzo que los hogares con jefatura masculina para obtener ingresos, para asegurar que sus hijos concluyan la educación primaria, y tener viviendas con servicios básicos y pisos de cemento o loseta. Asimismo, debemos considerar que estos hogares sin esposo reciben ayudas monetarias y en especie de familiares, compadres, amigos y de los programas de gobierno (PROCAMPO y OPORTUNIDADES). ${ }^{8}$ Las estrategias de estos hogares para alcanzar sus metas de bienestar están asociadas a su menor tamaño y a la migración de sus miembros tanto interna como internacional (ver el coeficiente de correlación correspondiente en el cuadro 2). La solidaridad familiar y comunitaria así como las estrategias de estos hogares para incrementar sus ingresos ayudan a reducir su vulnerabilidad social. Estos resultados coinciden con aquellos reportados en otros trabajos (González de la Roca, 1997; Reyes Morales, 2004). En cambio, no existe una correlación significativa entre la falta de acceso a los servicios públicos y privados de salud o entre el analfabetismo y alguno de los dos tipos de hogares. Esto puede estar asociado al hecho de que la gran mayoría de los oaxaqueños no tiene acceso a la salud (85.3\%) y el analfabetismo seguramente se encuentra asociado a los bajos ingresos y a la vulnerabilidad de la economía campesina. En nuestra opinión, los servicios de salud junto con el analfabetismo constituyen las mayores deudas sociales del Estado con la población pobre de México y no tanto el que se cuente con pisos de cemento o con vivienda sin hacinamiento.

\section{Vulnerabilidad de la población indígena y de las mujeres indígenas}

A diferencia de los hogares con jefatura femenina, la población indígena guarda una correlación directa con el índice de marginación. Este tipo de correlación se mantiene para sus componentes y en particular conviene subrayar la correlación con la población de bajo poder adquisitivo, con la población en localidades cuyo equipamiento urbano es normalmente deficiente, con la población analfabeta tanto femenina como masculina y con el índice de vivienda (cuadro 3). La correlación es inversa con el grado promedio de escolaridad para ambos sexos y confirma la correlación directa con el analfabetismo. De esta manera, se observa la grave marginación de la población indígena sin distinción de sexos. La población indígena en Oaxaca se encuentra asociada estrechamente con el analfabetismo, con un bajo grado promedio de escolaridad, bajo poder adquisitivo, deficiente equipamiento urbano y vivienda precaria. Con estas carencias, la población indígena se coloca en una clara condición de exclusión de las políticas de bienestar y de los beneficios del modelo de desarrollo nacional. El índice de electrodomésticos que indica la posesión de aparatos electrodomésticos modernos - que normalmente son de consumo generalizado (televisor, refrigerador, lavadora y en menor grado la computadora personal) - está inversamente correlacionado con la población indígena. Este resultado comprueba el bajo poder adquisitivo de la población indígena, cuyo ingreso se destina principalmente a la subsistencia. La situación de la población indígena es aún más grave si consideramos que ésta guarda una correlación directa con la población sin derechohabiencia a servicios de salud. Paradójicamente, dicha correlación 
no es estadísticamente significativa para los hogares con jefatura femenina, en los que la literatura sociológica norteamericana pone especial énfasis, ${ }^{9}$ ni para los hogares con jefatura masculina. Esto indica que en, materia de salud, educación y servicios de la vivienda la población indígena es el sector de la población excluido de las políticas públicas. Por consiguiente, la población indígena es el grupo de la población oaxaqueña más vulnerable a las catástrofes naturales, a las enfermedades y las crisis económicas. Por su baja escolaridad, los indígenas se encuentran en clara desventaja para poder competir por salarios bien remunerados y, además, son víctimas de discriminación.

La población indígena se caracteriza por tener hogares más grandes (correlación directa) y por participar menos en la migración interna de mujeres y hombres que el resto de la población (correlación inversa). La migración internacional no guarda una correlación estadísticamente significativa con la población indígena. El tamaño del hogar muestra otra dimensión de la vulnerabilidad de los hogares indígenas, ya que al tener más miembros los hogares aumentan su nivel de precariedady, por lo tanto, se encuentran prácticamente impedidos para aprovechar los servicios de educación pública y pagar servicios médicos particulares. Al parecer, la migración no constituye una fuente importante de ingreso para las familias indígenas que permanecen en sus comunidades.

La población indígena femenina guarda una correlación directa con el analfabetismo y una correlación inversa con el grado promedio de escolaridad. La población indígena masculina también presenta el mismo tipo de correlación para estas variables, pero el valor absoluto de su coeficiente de correlación es menor que el de la población indígena femenina. Estos resultados que aparecen en el cuadro tres y aquellos del cuadro uno confirman que las mujeres indígenas dentro de la población indígena representan el sector con mayor exclusión de la política pública de educación. Sin embargo, los cuadros uno, dos y tres muestran que la población indígena tiene menos migración y en particular la población indígena femenina. Esta situación se relaciona con los bajos ingresos de la población indígena y con el asilamiento de gran mayoría de los municipios indígenas.

\section{San Francisco, una comunidad indígena zapoteca de alta intensidad migratoria y con alta escolaridad}

Los resultados de una investigación realizada entre 2004 y 2006 en San Francisco Cajonos, ${ }^{10}$ una comunidad indígena zapoteca de alta intensidad migratoria permiten analizar las tendencias de largo plazo de la vulnerabilidad en las mujeres indígenas y mestizas. Los habitantes de esta comunidad han logrado conservar por tradición oral durante siglos o quizás milenios la historia de su origen como pueblo. San Francisco Cajonos aparece durante el período colonial como piedra angular de la cristianización del distrito de Villa Alta en la región Sierra Norte de Oaxaca y durante la Revolución mexicana es víctima de la lucha armada entre facciones.

San Francisco Cajonos se involucró en la migración internacional desde que se inició el 'programa bracero' a principios de los años cuarenta del siglo XX. La migración interna se intensificó a partir de 1960 cuando se construyó la primera carretera que conectó esta comunidad con la ciudad de Oaxaca. Uno de los efectos visibles de la migración en la estructura poblacional ha sido un marcado desequilibrio en la población total por sexos. La población femenina constituye 55.6\% de la población total local. Entre la población de migrantes internos la población femenina es aún mayor (62\%). En cambio, entre los migrantes internacionales la población femenina es menos de la mitad de la población total (47.5\%). ${ }^{11}$ Otro efecto de la migración es la reducción permanente de la población total a partir de 1960. El tamaño del hogar se ha mantenido alrededor de tres miembros, en promedio, entre 1990 y 2005, mientras que en el área metropolitana de Los Ángeles, California, los hogares de los migrantes tienen cuatro miembros en promedio. ${ }^{12}$ La migración también ha permitido que la cultura de esta comunidad 
llegue a las ciudades de Oaxaca, México y Los Ángeles a través de los clubes de los migrantes.

Un análisis de la estructura de edades, en 2005, apoyado con entrevistas a profundidad, muestra que las mujeres estaban marginadas de la educación formal al iniciarse el proceso migratorio y hasta la construcción de la primera carretera (Reyes Morales y Gijón Cruz, 2005). En el cuadro cuatro se puede ver que el analfabetismo aún subsiste en la población local y en la población femenina es cinco veces mayor que en la población masculina. Sin embargo, el analfabetismo femenino de la comunidad de estudio es aproximadamente la mitad del que se presenta en la entidad (comparar cuadros 1 y 4). En realidad, el analfabetismo es un vestigio del pasado que se concentra principalmente en la tercera edad, mientras que entre los migrantes internos e internacionales prácticamente ha desaparecido. Los migrantes internacionales han puesto más énfasis en la educación básica, la secundaria y la media básica que los migrantes internos y la comunidad de origen. Los migrantes internos han centrado su atención en la educación de la mujer a partir de la secundaria, superando a los migrantes internacionales. Este esfuerzo centrado en las mujeres está relacionado con el desequilibrio tan marcado de la estructura poblacional por sexos; como podemos ver en el cuadro cuatro los hombres también destacan en la educación universitaria. En San Francisco Cajonos también se realizan esfuerzos para elevar la escolaridad en todas las esferas pero sobre todo, destaca la educación primaria.

En este estudio de caso es claro el papel de la migración en el cambio de actitud de los jefes del hogar hacia la educación de las mujeres, quienes se han convertido en sujetos activos de la economía familiar tanto en la comunidad de origen como en los lugares de destino. En parte, esto tiene que ver con el desequilibrio demográfico inducido por la migración y en parte por la necesidad de obtener mayores ingresos para elevar la calidad de vida de los hogares. Es decir, la inversión en la educación de la mujer es el resultado de la adaptación al nuevo contexto demográfico en la búsqueda de mejores condiciones de vida.

Así, al principio del nuevo milenio la comunidad de estudio aparece como una comunidad culta y dinámica en los escenarios estatales, nacionales e internacionales. Un ciudadano de San Francisco Cajonos ocupó la presidencia de la Comisión de Derechos Humanos el Senado de la República; ${ }^{13}$ los niños representan a su región cultural en la guelaguetza infantil, en la ciudad de Oaxaca; y los migrantes rescatan templos católicos abandonados en Los Ángeles, California. Las mujeres migrantes llevan la batuta en captación y envío de las remesas colectivas para la fiesta y la construcción del templo de "Los Mártires de Cajonos", personajes del siglo XVIII que fueron beatificados por el Papa Juan Pablo II el I de agosto de 2002. En Los Ángeles, no faltan las mujeres empresarias de San Francisco Cajonos que organizan el mercado laboral, en el área de los servicios de limpieza, para sus paisanas recién llegadas. Los hombres tienen una activa participación en la política en la región de influencia de San Francisco Cajonos (sector Cajonos) y en los lugares de destino a través de sus clubes de migrantes. La participación política ha llegado incluso al enfrentamiento con el gobierno del estado de Oaxaca, como ocurrió durante 2006. Los clubes de migrantes de los municipios del sector Cajonos, radicados en el estado de California, apoyaron al movimiento social de la sección 22-APPO, y presionaron a sus autoridades municipales -incluidas las de la comunidad de estudio- para participar en las marchas y apoyar económicamente a sus maestros en los plantones.

La migración y sus consecuencias demográficas, en la búsqueda de mejores condiciones de vida, aparecen como factores que han influido en los cambios de roles de las mujeres en San Francisco Cajonos. Las mujeres han pasado de ser amas de casas sumisas y analfabetas a sujetos sociales trasnacionales, educados y emprendedores. La educación y la migración - junto con una inusual capacidad de gestión que caracteriza a los municipios de la región Sierra Norte— han sido sin duda 
los principales medios de movilidad social. Un grado de escolaridad permite, en general, acceder a mejores salarios y, además, adquirir prestigio social. La migración es el medio que permite obtener empleos mejor remunerados en los mercados laborales nacionales y de los Estados Unidos.

En la comunidad de origen, la educación básica llega hasta la primaria, y los adolescentes asisten a la secundaria en San Pedro Cajonos, a cuatro kilómetros de distancia. Por lo general, los jóvenes continúan su educación media superior y superior en la zona metropolitana de la ciudad de Oaxaca, aprovechando las remesas y subsidios de gobierno. La comunidad cuenta con una clínica de la Secretaría de Salud y una carretera recién pavimentada. La mayor parte de las calles están pavimentadas y existe alumbrado público. Las viviendas disponen de agua entubada, drenaje y electrificación como resultado de la gestión del gobierno local ante los gobiernos estatal y federal, de la coalición de gobiernos locales del sector Cajonos pero también del flujo de las remesas colectivas. Aquí, la lección aprendida es que los grupos vulnerables no son sujetos pasivos e indefensos que sucumben ante las fuerzas del libre mercado y que son abandonados a su suerte por el Estado mexicano insensible a los problemas sociales de los pueblos indígenas. Las mujeres indígenas pueden salir de la pobreza y liberarse del yugo familiar que las excluye de la educación cuando las metas familiares son aquellas de mejorar las condiciones de vida través de la migración. En este sentido, la migración se convierte en un instrumento de transformación social al margen del Estado mexicano y sin recurrir a movimientos sociales violentos. La migración se ha convertido en una estrategia familiar para vencer la pobreza en la que seguirían sumidos los miembros del hogar esperando apoyo de un gobierno central indiferente al destino de la gran masa pobre del país. Sin migración, la educación sería el principal instrumento de movilidad social que permitiría a los pobres salir de su precariedad y revalorarse socialmente, ya sea en sus comunidades de origen o en los lugares de destino.

\section{Conclusiones}

Los hallazgos en los municipios del estado de Oaxaca, en 2005, contradicen la situación de precariedad relativa de los hogares con jefatura femenina reportada por la literatura especializada (González de la Rocha, 1997; Reyes Morales, 2004). La respuesta se encuentra asociada a las estrategias que han adoptado estos hogares para mejorar su nivel de bienestar: 1) un menor tamaño de los hogares; 2) mayor énfasis en la educación; 3) la migración como fuente de ingresos; y 4) una mayor preferencia para asentarse en localidades con mayor equipamiento urbano y mejor acceso a las políticas públicas de bienestar (educación, servicios de la vivienda y salud). Las tres primeras coinciden con el estudio de caso que agrega la identidad indígena como un recurso que permite aglutinar esfuerzos individuales en pro de la comunidad de origen y de las comunidades de migrantes.

La población indígena sigue siendo el grupo excluido de las políticas públicas de bienestar y en particular la mujer indígena concentra el analfabetismo y los niveles más bajos de escolaridad. Esta situación puede también estar ligada a razones de orden cultural de las sociedades indígenas y los pobres urbanos pero también estar ligada al aislamiento de las comunidades indígenas. La migración y las carreteras rompen el aislamiento de las sociedades rurales y cambian los roles de las mujeres indígenas (Gijón Cruz y Reyes Morales, 2007).

El conteo de población y vivienda 2005 muestra que en materia de salud, en el estado de Oaxaca, ocurre el mayor rezago de las políticas de bienestar. La gran mayoría de la población se encuentra en situación de riesgo ante epidemias y otras contingencias debido su bajo poder adquisitivo (cuadro 1). Esta situación explica por qué Oaxaca se encuentra en el fondo de estadísticas de mortalidad. La experiencia de San Francisco Cajonos muestra que la gestión del gobierno local, junto con el apoyo de los clubes de migrantes, ofrece instrumentos capaces de llamar la atención de los gobiernos estatal y federal hacia la provisión de servicios básicos de vivienda, 
equipamiento urbano, escuelas y servicios de salud. De esta manera, las políticas públicas se convierten en herramientas sociales que permiten mejorar las condiciones de vida de los grupos más vulnerables.

Otro hallazgo que conviene resaltar se refiere a los avances en la posesión de electrodomésticos, como televisor y refrigerador, los cuales son visibles aun entre la población indígena. En menor grado, es visible la presencia de lavadoras de ropa. En cambio, la computadora está apenas por arriba de un punto porcentual en los hogares con jefatura masculina y en la población indígena; llega a cuatro puntos porcentuales en los hogares con jefatura femenina. El proceso de modernización del país se encuentra en franca penetración en todos los rincones de la entidad y la escasez de liquidez se compensa con remesas y los subsidios de gobierno (PROCAMPO y OPORTUNIDADES).
Finalmente, podemos concluir que el grupo excluido de las políticas de bienestar y con menor consumo de aparatos electrodomésticos es la población indígena, mientras que las mujeres indígenas constituyen el grupo particularmente excluido de la política pública de educación. La solidaridad familiar y comunitaria, las estrategias de los hogares con jefatura femenina para obtener ingresos y recibir beneficios de las políticas públicas, los coloca en una situación más bien de ventaja que de desventaja frente los hogares con jefatura masculina. El modelo de desarrollo nacional, que pone el énfasis en el libre mercado y en la globalización, excluye de los beneficios de una de las economías más grandes del planeta a la gran masa pobre. En el caso particular del estado de Oaxaca, la precariedad y la exclusión de las políticas de bienestar estan concentradas principalmente en la población indígena.

Fig. 1 Porcentaje de población femenina por municipio, estado de Oaxaca, 2005.

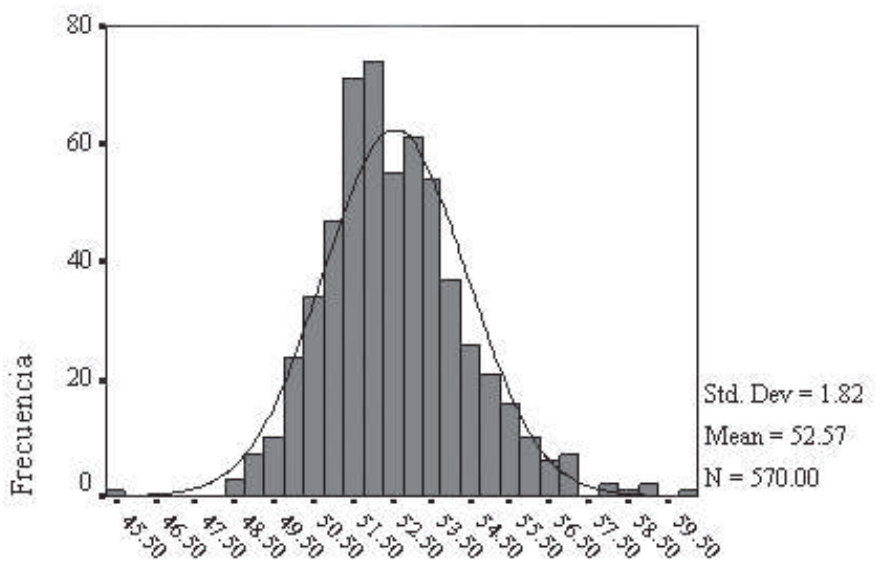

Porcentaje de población femenina 
Fig. 2 Porcentaje de población indígena por municipio, estado de Oaxaca, 2005.

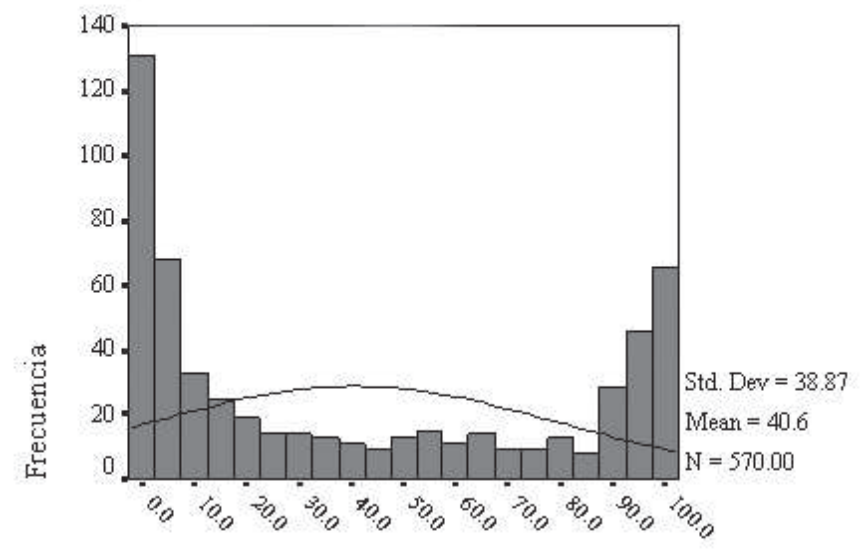

Porcentaje de población indigena por municipio

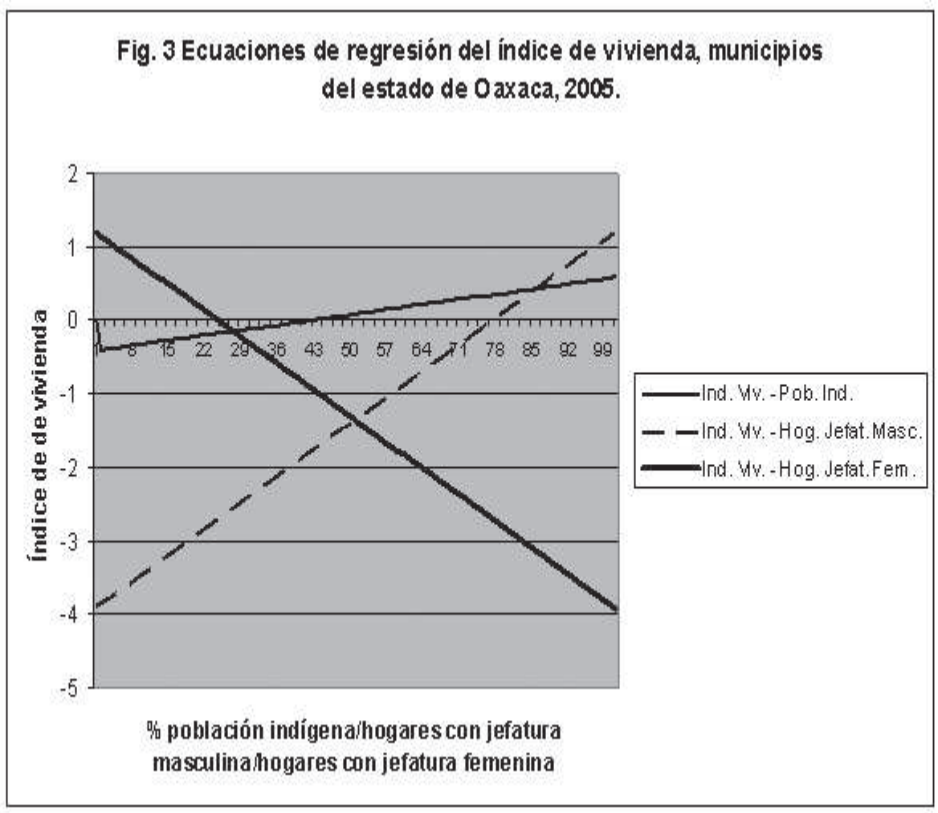




\section{Cuadro 1}

Población indígena, hogares con jefatura femenina y masculina en el estado de Oaxaca: Indicadores de exclusión de las políticas públicas, de características socioeconómicas y de migración, 2005

\begin{tabular}{|c|c|c|c|c|}
\hline & \multicolumn{3}{|c|}{ Promedio de los municipios considerados } & \multirow[b]{2}{*}{$\begin{array}{c}\text { Estado de } \\
\text { Oaxaca } \\
\mathrm{N}=\mathbf{5 7 0}\end{array}$} \\
\hline & $\begin{array}{c}\text { Municipios con po- } \\
\text { blación indigena }^{\text {a }} \\
\mathrm{N}=245 \\
\end{array}$ & $\begin{array}{c}\text { Hogares con je- } \\
\text { fatura femenina }^{b} \\
\mathbf{N}=\mathbf{2 7 8} \\
\end{array}$ & $\begin{array}{c}\text { Hogares con je- } \\
\text { fatura masculina } \\
\mathrm{N}=292 \\
\end{array}$ & \\
\hline Porcentaje de población indígena de 5 años y más. & - & 33.21 & 47.35 & 35.2 \\
\hline Índice de marginación y grado de marginación. ${ }^{\text {d }}$ & $\begin{array}{c}1.09863 \\
\text { (Muy alto) }\end{array}$ & $\begin{array}{c}0.38676 \\
\text { (Alto) }\end{array}$ & $\begin{array}{c}0.86971 \\
\text { (Alto) }\end{array}$ & $\begin{array}{r}2.13041 \\
\text { (Muy alto) } \\
\end{array}$ \\
\hline $\begin{array}{l}\text { Población de bajo poder adquisitivo (porcentaje de población } \\
\text { ocupada con ingreso de hasta dos salarios mínimos). }\end{array}$ & 85.79 & 78.58 & 84.07 & 69.98 \\
\hline Índice de vivienda y nivel. ${ }^{\mathrm{e}}$ & $\begin{array}{c}0.40677 \\
\text { (Bajo) } \\
\end{array}$ & $\begin{array}{l}-0.28871 \\
\text { (Medio) } \\
\end{array}$ & $\begin{array}{c}0.27487 \\
\text { (Bajo) } \\
\end{array}$ & $\begin{array}{c}0.00000 \\
\text { (Medio) }\end{array}$ \\
\hline $\begin{array}{l}\text { Población en localidades con equipamiento urbano escaso (\% } \\
\text { de población en localidades con menos de } 5000 \text { habitantes). }\end{array}$ & 95.94 & 87.44 & 94.63 & 61.27 \\
\hline Porcentaje de población de 15 años y más analfabeta. & 29.42 & 21.47 & 23.86 & 19.35 \\
\hline Porcentaje de población femenina de 15 años y más analfabeta. & 36.42 & - & - & 12.62 \\
\hline Porcentaje de población masculina de 15 años y más analfabeta. & 21.09 & - & - & 6.70 \\
\hline Grado promedio de escolaridad de la población. & 4.53 & 5.47 & 4.98 & 6.39 \\
\hline Grado promedio de escolaridad de la población femenina. & 4.10 & - & - & 6.04 \\
\hline Grado promedio de escolaridad de la población masculina. & 5.02 & - & - & 6.79 \\
\hline Porcentaje de población sin derechohabiencia a servicios de salud. & 89.50 & 82.93 & 87.45 & 75.75 \\
\hline Índice de electrodomésticos y nivel. ${ }^{f}$ & $\begin{array}{l}-0.56139 \\
\text { (Bajo) } \\
\end{array}$ & $\begin{array}{l}0.28795 \\
\text { (Alto) }\end{array}$ & $\begin{array}{l}-0.27414 \\
\text { (Medio) } \\
\end{array}$ & $\begin{array}{c}0.00000 \\
\text { (Medio) }\end{array}$ \\
\hline $\begin{array}{l}\text { Tamaño del hogar (promedio de ocupantes en viviendas par- } \\
\text { ticulares habitadas). }\end{array}$ & 4.57 & 4.12 & 4.53 & 4.36 \\
\hline $\begin{array}{l}\text { Migración internacional (porcentaje de población de } 5 \text { años y } \\
\text { más residente en Estados Unidos en 2000). }\end{array}$ & 0.43 & 0.52 & 0.33 & 0.31 \\
\hline $\begin{array}{l}\text { Migración interna (porcentaje de población de } 5 \text { años y más } \\
\text { residente en otra entidad en 2000). }\end{array}$ & 1.13 & 1.84 & 1.21 & 1.71 \\
\hline $\begin{array}{l}\text { Migración interna femenina (porcentaje de población femeni- } \\
\text { na de } 5 \text { años y más residente en otra entidad en 2000). }\end{array}$ & 0.56 & - & - & 0.85 \\
\hline $\begin{array}{l}\text { Migración interna masculina (porcentaje de población mascu- } \\
\text { lina de } 5 \text { años y más residente en otra entidad en 2000). }\end{array}$ & 0.57 & - & - & 0.86 \\
\hline
\end{tabular}

${ }^{a}$ Los valores de las variables representan el promedio del valor correspondiente de los municipios cuya población indígena es mayor que el promedio del total de los municipios de la entidad (40.6\%).

${ }^{\mathrm{b}}$ Los valores de las variables representan el promedio del valor correspondiente de los municipios en donde el número de hogares con jefatura femenina es mayor que el promedio del total de los municipios de la entidad (23.6\%).

${ }^{c}$ Los valores de las variables representan el promedio del valor correspondiente de los municipios en donde el número de hogares con jefatura masculina es mayor que el promedio del total de los municipios de la entidad (76.4\%).

d Se tomó como base la estratificación del índice de marginación del país.

'Este índice se calculó mediante el método de componentes principales a partir de indicadores referidos a ocupantes en viviendas: sin drenaje ni servicio sanitario, sin energía eléctrica, sin agua entubada y con pisos de tierra. El índice tiene $81.482 \%$ de la variación total, utiliza un solo factor. Se establecieron cinco niveles o estratos, se aplicó el método de estratificación óptima de Danelius y Hodges. El valor del índice de vivienda para la entidad se estimó calculando el promedio de todos los municipios.

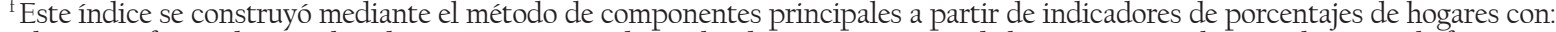
televisor, refrigerador, con lavadora y con computadora. El índice tiene $45.075 \%$ de la variación total y se utiliza un solo factor. Se establecieron cinco niveles o estratos, se aplicó el método de estratificación óptima de Danelius y Hodges. El valor del índice de electrodomésticos para la entidad se estimó calculando el promedio de todos los municipios.

Fuente: CONAPO, 2006, Indices de marginación, 2005; INEGI, 2006, Conteo general de población y vivienda, 2005. 


\section{Cuadro 2}

Coeficientes de correlación de Pearson entre los hogares con jefatura femenina y masculina e indicadores socioeconómicos y de migración, municipios del estado de Oaxaca, 2005

$$
\mathbf{N}=\mathbf{5 7 0}
$$

\begin{tabular}{|c|c|c|c|}
\hline & \begin{tabular}{|c|}
$\begin{array}{c}\text { Porcentaje de } \\
\text { hogares con jefatura } \\
\text { femenina }\end{array}$ \\
\end{tabular} & \begin{tabular}{|c|}
$\begin{array}{c}\text { Porcentaje de } \\
\text { hogares con jefatura } \\
\text { masculina }\end{array}$ \\
\end{tabular} & $\begin{array}{c}\text { Porcentaje de } \\
\text { población femenina }\end{array}$ \\
\hline Porcentaje de población indígena de 5 años y más. & $-0.196 * *$ & $0.196 * *$ & $0.005 n s$ \\
\hline IIndice de marginación. & $-0.296 * *$ & $0.296 * *$ & $-0.148 * *$ \\
\hline Porcentaje de población de 15 años y más analfabeta. & $-0.079 n s$ & $0.079 n s$ & $-0.002 n s$ \\
\hline Grado promedio de escolaridad. & $0.152 * *$ & $-0.152 * *$ & $-0.010 n s$ \\
\hline IIndice de vivienda. ${ }^{a}$ & $-0.318 * *$ & $0.318 * *$ & $-0.198 * *$ \\
\hline $\begin{array}{l}\text { Población de bajo poder adquisitivo (porcentaje de } \\
\text { población ocupada con ingresos de hasta dos salarios } \\
\text { mínimos). }\end{array}$ & $-0.194 * *$ & $0.194 * *$ & $-0.041 \mathrm{~ns}$ \\
\hline IIndice de electrodomésticos. & $0.254 * *$ & $-0.254 * *$ & $0.108 * *$ \\
\hline $\begin{array}{l}\text { Porcentaje de población sin derechohabiencia a servi- } \\
\text { cios de salud. }\end{array}$ & $0.937 \mathrm{~ns}$ & $0.937 \mathrm{~ns}$ & $0.027 \mathrm{~ns}$ \\
\hline $\begin{array}{l}\text { Tamaño del hogar (promedio de ocupantes en vivien- } \\
\text { das particulares habitadas). }\end{array}$ & $-0.400 * *$ & $0.400 * *$ & $-0.259 * *$ \\
\hline $\begin{array}{l}\text { Migración internacional (porcentaje de población de } 5 \\
\text { años y más residente en Estados Unidos en 2000). }\end{array}$ & $0.168 * *$ & $-0.168 * *$ & $0.194 * *$ \\
\hline $\begin{array}{l}\text { Migración interna (porcentaje de población de } 5 \text { años y } \\
\text { más residente en otra entidad en 2000). }\end{array}$ & $0.204 * *$ & $-0.204 * *$ & $0.089 *$ \\
\hline
\end{tabular}

** La correlación es estadísticamente significativa para p < 0.01 (prueba de dos colas)

* La correlación es estadísticamente significativa para p < 0.05 (prueba de dos colas)

ns No significativa.

a En la escala de este índice, los valores más negativos están asociados a la categoría de muy alto, mientras que los valores positivos más altos están asociados a la categoría de muy bajo. Así, el signo negativo del coeficiente de correlación indica una relación directa entre las variables consideradas.

Fuente: CONAPO, 2006, Índices de marginación, 2005; INEGI, 2006, Conteo general de población y vivienda, 2005. 


\section{Cuadro 3}

Coeficientes de correlación de Pearson entre la población indígena e indicadores socioeconómicos y de migración, municipios del estado de Oaxaca, 2005

$\mathbf{N}=\mathbf{5 7 0}$

\begin{tabular}{|c|c|c|c|}
\hline & \begin{tabular}{|c|} 
Porcentaje de \\
población indigena
\end{tabular} & & \begin{tabular}{|c|} 
Porcentaje de \\
población indigena
\end{tabular} \\
\hline Índice marginación & $0.510 * *$ & \begin{tabular}{|l|} 
Población de bajo poder adquisitivo (porcentaje de población \\
ocupada con ingreso de hasta dos salarios mínimos).
\end{tabular} & $0.338 * *$ \\
\hline $\begin{array}{l}\text { Población en localidades con equipamiento urbano } \\
\text { escaso (porcentaje de población en localidades con } \\
\text { menos de } 5000 \text { habitantes). }\end{array}$ & $0.154 * *$ & Índice de electrodomésticos. & $-0.520 * *$ \\
\hline Índice de vivienda & $0.387 * *$ & $\begin{array}{l}\text { Porcentaje de población sin derechohabiencia a servicios } \\
\text { de salud. }\end{array}$ & $0.211 * *$ \\
\hline $\begin{array}{l}\text { Porcentaje de población femenina de } 15 \text { años y } \\
\text { mas analfabeta. }\end{array}$ & $0.573 * *$ & $\begin{array}{l}\text { Tamaño del hogar (promedio de ocupantes en viviendas } \\
\text { particulares habitadas) }\end{array}$ & $0.322 * *$ \\
\hline $\begin{array}{l}\text { Porcentaje de población masculina de } 15 \text { años y } \\
\text { mas analfabeta. }\end{array}$ & $0.448 * *$ & \begin{tabular}{|l} 
Migración internacional (porcentaje de población de 5 años \\
y más residente en Estados Unidos en 2000).
\end{tabular} & $0.008 \mathrm{~ns}$ \\
\hline $\begin{array}{l}\text { Grado promedio de escolaridad de la población } \\
\text { femenina. }\end{array}$ & $-0.487 * *$ & \begin{tabular}{|l} 
Migración interna femenina (porcentaje de población fe- \\
menina de 5 años y más residente en otra entidad en 2000).
\end{tabular} & $-0.231 * *$ \\
\hline $\begin{array}{l}\text { Grado promedio de escolaridad de la población } \\
\text { masculina. }\end{array}$ & $-0.381 * *$ & $\begin{array}{l}\text { Migración interna masculina (porcentaje de población mas- } \\
\text { culina de } 5 \text { años y más residente en otra entidad en 2000). }\end{array}$ & $-0.215 * *$ \\
\hline
\end{tabular}

** La correlación es estadísticamente significativa para p < 0.01 (prueba de dos colas). ns: No significativa.

Fuente: CONAPO, 2006, Índices de marginación, 2005; INEGI, 2006, Conteo general de población y vivienda, 2005.

Cálculos propios en el programa SPSS para Windows.

\section{Cuadro 4}

Escolaridad de la población local, de los migrantes internos y migrantes internacionales en San Francisco Cajonos, 2005

\begin{tabular}{|c|c|c|c|c|c|c|}
\hline & \multicolumn{3}{|c|}{ Población masculina } & \multicolumn{3}{|c|}{ Población femenina } \\
\hline & Local & $\begin{array}{l}\text { Migrantes } \\
\text { internos }\end{array}$ & $\begin{array}{c}\text { Migrantes } \\
\text { internacionales }\end{array}$ & Local & $\begin{array}{l}\text { Migrantes } \\
\text { internos }\end{array}$ & $\begin{array}{c}\text { Migrantes } \\
\text { internacionales }\end{array}$ \\
\hline Analfabeta. & 2.1 & 0.0 & 0.9 & 10.7 & 0.8 & 0.0 \\
\hline 1-6 años de escolaridad (primaria). & 30.8 & 16.8 & 26.9 & 30.8 & 24.0 & 20.4 \\
\hline 7-9 años de escolaridad (secundaria). & 6.0 & 10.4 & 17.6 & 6.8 & 9.6 & 10.2 \\
\hline 10-12 años de escolaridad (bachillerato). & 3.4 & 3.2 & 4.6 & 4.3 & 8.8 & 11.1 \\
\hline $\begin{array}{l}\text { 13-18 años de escolaridad (carrera univer- } \\
\text { sitaria o normalista). }\end{array}$ & 2.6 & 8.0 & 4.6 & 2.6 & 18.4 & 3.7 \\
\hline Total & 44.9 & 38.4 & 54.6 & 55.1 & 61.6 & 45.4 \\
\hline
\end{tabular}

Fuente: Censo de población y migración, 2005, proyecto "Comunidades transnacionales México-Estados Unidos. Caso Oaxaca (Regiones: Valles Centrales y Sierra Norte)", financiado por la Fundación Rockefeller, 2004-2006. 


\section{Notas}

${ }^{1}$ Ver Nourisis, Marija J. SPSS for Windows: Base Systems User's Guide, Release 6.0.

${ }^{2}$ CONAPO, Proyecciones de la población de México, 2000-2030, México, 2002.

${ }^{3}$ Estimaciones del CONAPO, con base en Proyecciones de la Población de México, 2000-2030.

${ }^{4}$ Estimaciones del CONAPO, con base en Proyecciones de la Población de México, 2000-2030.

${ }^{5}$ CONAPO, 2006, Índices de marginación por municipio, 2005, México. El índice de marginación considera estos indicadores de la siguiente manera: el porcentaje de habitantes en viviendas sin agua entubada, sin drenaje ni servicio sanitario, sin energía eléctrica y con pisos de tierra; el porcentaje de la población de 15 años y más analfabeta y sin primaria completa; el porcentaje de población ocupada que gana hasta dos salarios mínimos; y porcentaje de población en localidades de menos de 5000 habitantes. Asimismo, incluye porcentaje de viviendas con algún nivel de hacinamiento, indicador que no fue considerado porque si bien permite identificar características y estándares de las viviendas urbanas, éste no forma parte de las políticas públicas para el medio rural.

${ }^{6}$ Es conveniente aclarar que el índice de marginación por estados no tiene la misma escala que en el ámbito municipal; por consiguiente, la comparación del índice de marginación entre municipios y estados no es válida, aunque el grado de marginación se estratifica siguiendo el mismo criterio.

${ }^{7}$ Reyes Morales, Rafael G., 2005, Condicionantes del bienestar en los hogares pobres de Oaxaca, Bode, Marilyn Eichner and Earl W. Morris, "The role of constraints in housing adjustment", en Edward G. Goetz, Sehwa Yang Khil and Earl W. Morris (eds.), Social science research on housing, p.15, Morris, Earl W. and Winter, Mary, Housing, family and society, p. 61 y 66, González de la Rocha, Mercedes, "Family well-being, food consumption, and survival strategies during Mexicós economic crisis", en González de la Rocha, M. and Escobar Latapí, A. (eds.), Social responses to Mexicós economic crisis, pp.121-125, González de la Rocha, Mercedes, "Hogares con jefatura femenina en México: Patrones y formas de vida”, Lázaro Castellanos, Rosa, Emma Zapata Martelo, Beatriz Martínez Corona y Pilar Alberti Manzanares, "Jefatura femenina de hogar y transformaciones en los modelos de género tradicionales en dos municipios de Guanajauato", Revista La Ventana, núm. 22.

${ }^{8}$ Se ha detectado esta situación a través de proyectos e investigaciones en las regiones Mixteca, Valles Centrales y Sierra Norte "OAXACALIFORNIA: Research and Initiation of Remittance Based Financial Mechanisms for Community Development in Migrant Sending Regions of México", 1999-2000, UC Mexus-CONACYT; ("El uso de las remesas internacionales y el papel de las organizaciones binacionales en el desarrollo de la mixteca oaxaqueña”, 2000-2002, SIBEJ, clave 19990503012; "Globalization and public goods from below: Migrant organisations, productive remittances, and economic development between México and California”, 2001-2002, Grant \#00-67264-002-GSS, Fundación MacArthur).

${ }^{9}$ Morris 1994a, Morris, p.15, Earl W, and Winter, Mary. Housing, family and society, p. 61 y 66, Coveney, Anne R. and Nancy Rudd, "Determinants of housing satisfaction of low-income, rural, male family heads"; Morris, Earl W., "Household, kin and nonkin sources of assistance in home building: The case of Oaxaca city", Urban Anthropology, Vol. 20(1), 49-66.

${ }^{10}$ Como parte de esta investigación se aplicaron dos diferentes encuestas (la etnoencuesta del Mexican Migración Project y la encuesta para obtención de la matriz de Contabilidad social); se realizó un censo de población y migración, entrevistas a profundidad a migrantes que participaron en el programa bracero, observación participante y videos de las fiestas patronales.

11 "Censo de población y migración de San Francisco Cajonos, 2005", proyecto "Comunidades transnacionales México-Estados Unidos. Caso Oaxaca (Regiones: Valles Centrales y Sierra Norte)", financiado por la Fundación Rockefeller, 2004-2006. 
12 "Encuesta a migrantes de San Francisco Cajonos, Oaxaca en Los Angeles, California, 2005", proyecto "Comunidades transnacionales México-Estados Unidos. Caso Oaxaca (Regiones: Valles Centrales y Sierra Norte)", financiado por la Fundación Rockefeller, 2004-2006.

${ }^{13}$ Fue electo al cargo de senador por el estado de Oaxaca durante LIX legislatura, 2003-2006.

\section{Bibliografía}

Alvarez Ayuso, Inmaculada y Edel Cadena Vargas, 2006, "Índice de vulnerabilidad social en los países de la OCDE”, Quievera, julio-diciembre, vol. 8, núm. 2, Universidad del estado de México, Toluca, México, pp. 248-274.

Bode, Marilyn Eichner and Earl W. Morris, 1994, "The role of constraints in housing adjustment", en Edward G. Goetz, Sehwa Yang Khil and Earl W. Morris (eds.), Social science research on housing, vol. II. University of Minnesota, Hosing Programa, Department of Desgn Housing and Apparel.

Busso, Roberto, 2001, "Vulnerabilidad social: nociones e implicancias de políticas para Latinoamerica a inicios del siglo XXI", Seminario internacional "Las diferentes expresiones de la vulnerabilidad social en América Latina y el Caribe”, CEPAL, Santiago de Chile, 20 y 21 de junio.

CONAPO, 2006, Índices de marginación 2005.

Cortés, Feranando, 2006, Consideraciones sobre la marginación, la marginalidad, la marginación económica y la exclusión social, Papeles de población, eneromarzo, núm. 47, Universidad del estado de México, Toluca, México, pp. 71-84.

Coveney, Anne R., and Nancy Rudd, 1986, "Determinants of housing satisfaction of low-income, rural, male family heads", Housing and Society, vol. 13, núm. 1, pp.3-18.

Damill, Mario, Roberto Frenkel y Roxana Mauricio, 2003, Políticas macroeconómicas y vulnerabilidad social, La Argentina en los años noventa, Serie Financiamiento del Desarrollo, núm. 135, CEPAL, Santiago de Chile.
Gijón Cruz, Alicia Sylvia and Rafael Reyes Morales, 2007, "International migration and changes in women's roles in indigenous communities, Oaxaca, Mexico", Tercera Cumbre de las Planicies: Inmigración y Cambio en Nuestras Comunidades, 26 al 29 de abril, Omaha, Nebraska, Estados Unidos, http://www.unomaha.edu/ ollas/papers.php.

González de la Rocha, Mercedes, 1991, "Family well-being, food consumption, and survival strategies during Mexicós economic crisis", en González de la Rocha, M., and Escobar Latapí, A. (eds.), Social responses to Mexicós economic crisis, San Diego, California, Center for U.S., Mexican Studies, University of California, pp.115-127.

—, 1997, "Hogares con jefatura femenina en México: Patrones y formas de vida”, Ponencia presentada al XX Congreso Internacional de la Sociedad de Estudios Latinoamericanos (ALAS), Guadalajara, México, 17-19 de abril.

Kaztman, Rubén, 2000, "Notas sobre la medición de la vulnerabilidad social”, documento presentado al Qunito Taller Regional "La medición de la Pobreza: métodos y aplicaciones” (LC/R.2026), CEPAL, IDEC, 6 al 8 de junio, Santiago de Chile.

Lázaro Castellanos, Rosa; Emma Zapata Martelo; Beatriz Martínez Corona y Pilar Alberti Manzanares, 2005, "Jefatura femenina de hogar y transformaciones en los modelos de género tradicionales en dos municipios de Guanajauato", La Ventana, núm. 22, pp.219-268.

Martínez Alonso, Libia Constanza, 2004, "Revisión del modelo de estratificación socioeconómica aplicado en Bogotá D.C.", en La estatificación en Bogotá D.C.y estudios relacionados, 1983-2004, Alcaldía Mayor de Bogotá, Departamento Administrativo de Planeación, Bogotá D.C., pp.60-90.

Morris, Earl W., 1991, "Household, kin and nonkin sources of assistance in home building: The case of Oaxaca city", Urban Anthropology, vol. 20(1): 49-66.

-, and Winter, Mary, 1996, Housing, family and society, Ames, Iowa, Earl W., Morris and Mary Winter. 
Nourisis, Marija J., 1993, SPSS for Windows: Base Systems User's Guide, Release 6.0.

Pizarro, Roberto, 2001, La vulnerabilidad social y sus desafíos: Una mirada desde América Latina, Serie Estudios Estadísticos Prospectivos, núm. 6, Naciones Unidas-CEPAL, Santiago de Chile.

Portes, Alejandro and Luis E. Guarnizo, 1991, "Tropical capitalists: U.S.-bound inmigration and small-enterprise development in the Dominican Republic", en Sergio Díaz-Brinquets and Sidney Weintraub (eds.), Migration, remittances, and small business development. Mexico and Caribbean Basin Countries, Westview Press, U.S.A.

Reyes Morales, Rafael G., 2004, Condicionantes del bienestar en los hogares pobres de Oaxaca, tesis doctoral, Instituto tecnológico de Oaxaca, Oaxaca, México.

-, et al., 2005, Remesas, proyectos productivos y desarrollo regional en el estado de Oaxaca, en Yolanda Massieu Trigo, Michelle Chauvet Sánchez \& Rodolfo García Zamora (coords. generales), Los actores sociales frente al desarrollo rural. Nueva ruralidad viejos problemas: 223-275, AMER y Editorial Praxis, México.

-, y Alicia Sylvia Gijón Cruz, 2005, Economía, Migración trasnacional, Cultura y Religión en San Francisco Cajonos, Instituto Tecnológico de Oaxaca, México, ISBN: 970-9916-02-5.

-, 2005, Economía, Migración trasnacional, Cultura y Religión en San Francisco Cajonos, Instituto Tecnológico de Oaxaca, México, ISBN: 970-9916-02-5.

Rodríguez Vignoli, Jorge, 2004, Vulnerabilidad social y sociodemográfica: distinciones conceptuales, antecedentes empíricos y aportes de política, Taller sobre vulnerabilidad NEPO, CELADE, 17 de febrero.

V. Nelly Salgado de Snyder, Tonatiuh González Vázquez, Ietza Bojorquez Chapela, César Infante Xibillé, 2007, "Vulnerabilidad social, salud y migración México-Estados Unidos", Salud Pública de México, vol. 49, p. 8-10, edición especial, XII congreso de investigación en salud pública, Instituto de Salud Pública, Cuernavaca, Morelos. 